\title{
Chapter 19 \\ Nonlinear Light Generation in Localized Fields Using Gases and Tailored Solids
}

\author{
Murat Sivis and Claus Ropers
}

\begin{abstract}
In Chap. 18, we demonstrated polarization-sensitive imaging at extremeultraviolet (EUV) wavelengths using a gas-phase high-harmonic generation (HHG) source. In a related project, we have investigated new types of gas-phase and solidstate EUV light sources employing field localization in plasmonic nanostructures and structured targets. Whereas our first results indicate that strong field confinement leads to exceedingly inefficient high-harmonic generation in gas-phase targets, for solid-state media efficient high-harmonic generation is possible in localized fields. The latter has great ramifications for new types of high-harmonic generation experiments and technological developments. Therefore, our research efforts aim in two directions: firstly, the development of new types of solid-state sources for highharmonic generation and, secondly, the application of locally generated solid-state high-harmonic signals for spectroscopy and imaging.
\end{abstract}

PACS Subject Classification: $42.65 . \mathrm{Ky} \cdot 81.07 .-\mathrm{b} \cdot 73.20 . \mathrm{Mf}$

\subsection{Plasmonic Enhancement for EUV Light Generation}

In this section, we discuss EUV light generation in gas-phase and solid targets using strong laser fields confined in plasmonic nanostructures. In the case of gas-phase targets, we critically revisit the feasibility of high-harmonic generation in resonant nanoantennas and tapered hollow waveguides (see Fig. 19.1 a and b), as reported previously [1, 2]. The results of our studies [3-5] show that for gas-phase targets, such as noble gas atoms, the measurable EUV emission exclusively originates from incoherent fluorescence instead of coherent high-harmonic radiation, which is due to an unfavorable conversion efficiency for coherent signals generated in low-density targets

M. Sivis · C. Ropers ( $\varangle)$

IV. Physical Institute - Solids and Nanostructures, Universität Göttingen,

Friedrich-Hund-Platz 1, 37077 Göttingen, Germany

e-mail: claus.ropers@uni-goettingen.de

M. Sivis

e-mail: murat.sivis@uni-goettingen.de

T. Salditt et al. (eds.), Nanoscale Photonic Imaging, Topics in Applied Physics 134, https://doi.org/10.1007/978-3-030-34413-9_19 

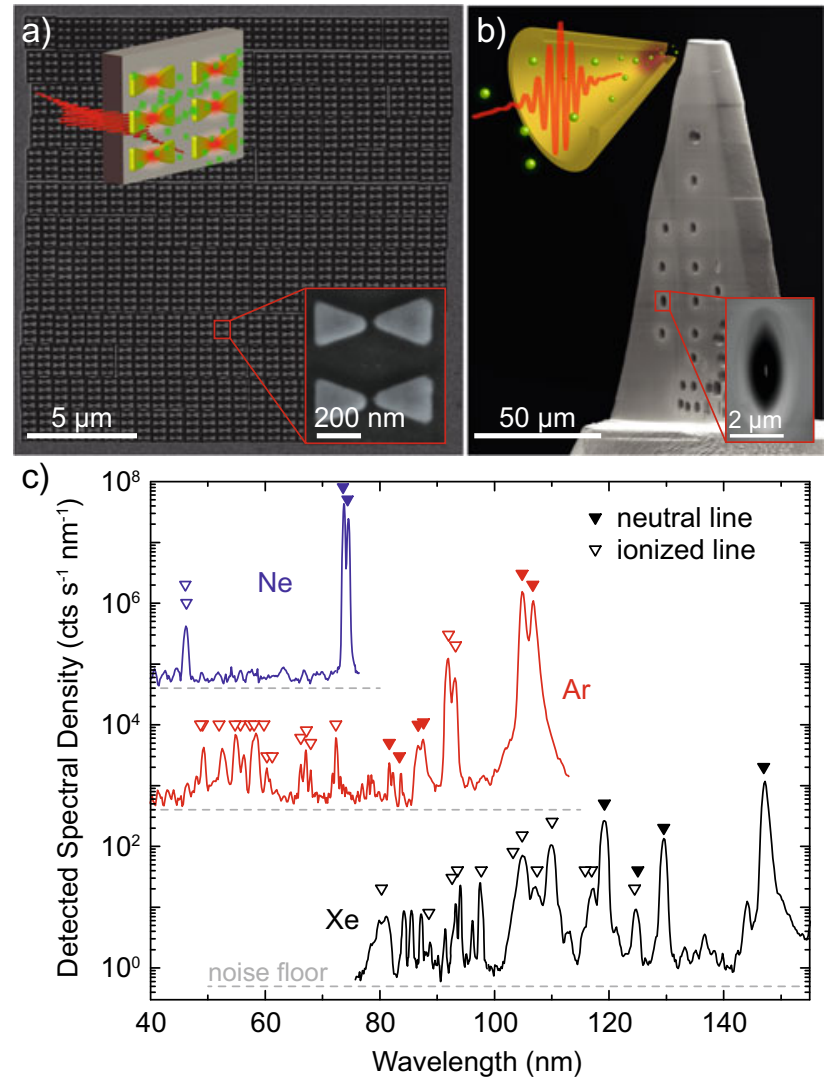

Fig. 19.1 Plasmon-enhanced EUV light generation. a Scanning electron micrograph of an array of gold bow-tie nanoantennas on a sapphire substrate. Lower inset: Close-up of two bow-tie antenna pairs. Upper inset: Schematic illustration of the gas excitation in the enhanced fields localized in the gap between the tips of the triangular antenna arms. b Scanning electron micrograph of a gold plateau containing a tapered hollow waveguide structure. Lower inset: Close-up of a waveguide, taken from the entrance aperture side. The waveguide has an entrance aperture size of several micrometers and tapers down to an exit aperture of few tens of nanometers in size. Upper inset: Schematic illustration of gas excitation in the locally enhanced field near the waveguide's exit aperture. c EUV spectra from xenon (Xe), argon (Ar) and neon (Ne), excited in the waveguide structure shown in (b). The argon and neon spectra are up-shifted to avoid overlap. Similar spectra have been observed from xenon and argon gas using bow-tie antennas for field enhancement

in nanometrically confined excitation volumes. After briefly describing our experiments and discussing the implications of the outcome, we highlight recent experiments, which overcome the limitations for plasmon-enhanced HHG by exchanging the gas-phase targets with solid-state materials.

In the experiment, the nanostructures are illuminated under vacuum conditions (pressure below $10^{-6}$ mbar) with low-energy, few-femtosecond laser pulses centered at $800 \mathrm{~nm}$ wavelength from a $78 \mathrm{MHz}$ Ti:sapphire oscillator. The incident laser field is 
confined in the particular structure leading to local intensities which are enhanced by more than two orders of magnitude compared to the incident intensity. Injected noble gas atoms (backing pressures up to 500 mbar) are excited and ionized in the enhanced field (see upper insets in Fig. 19.1a and b) via multiphoton absorption and strongfield excitation. The radiation emitted by the atoms is collected with an EUV flatfield spectrometer, which spectrally resolves the signal on an imaging microchannel plate detector. For both excitation schemes, the laser radiation is tightly focused to achieve incident intensities in excess of $0.1 \mathrm{TW} / \mathrm{cm}^{2}$. More detailed information on the experimental setup and conditions can be found elsewhere [3, 5].

Figure 19.1c shows a set of EUV fluorescence spectra obtained from different noble gases excited in a hollow waveguide structure, where all spectral features are identified with emission corresponding to electronic transitions of the respective gas species. The wavelength positions of the fluorescence lines are marked with full and open triangles for emission from neutral and singly ionized atoms, respectively. Similar spectra were measured using bow-tie nanostructures. The reason for the lack of coherent emission in these spectra can be found by considering the absolute conversion efficiency of the HHG process in the localized generation volume. The number of gas atoms in the generation volume is too small for a very efficient coherent build-up of the harmonic radiation. More specifically, the output power of (phasematched) high-harmonic generation scales quadratically with the pressure-length product, which is drastically reduced in implementations based on surface-plasmon field confinement in nanostructures. Since incoherent fluorescence scales only linearly with the pressure-length product, it can be efficiently generated and therefore is the predominant contribution to the EUV signal.

Estimates suggest that high-harmonic signal levels in plasmon-enhanced scenarios should be several orders of magnitude below that of the simultaneously emitted incoherent fluorescence $[5,6]$. Therefore, if possible at all, it will be very difficult to discriminate coherent from incoherent signals.

In a recent publication, the authors of the first studies on plasmon-enhanced gasphase high-harmonic generation $[1,2]$ report that a reproduction of their initial experiment resulted in the exclusive observation of incoherent emission and they acknowledge that their interpretation of the previous results in [1] was not fully correct, since they ignored the possibility of any incoherent contributions [7].

As described above, plasmon-enhanced high-harmonic generation in gaseous media is unfeasible under the given experimental conditions. However, the main limitation - the too low gas atom density in the generation volume-can be overcome by exchanging the gas with condensed matter targets. Reports on plasmon-enhanced high-harmonic generation in sapphire [5, 7], silicon [8], and zinc-oxide [9] crystals re-initiate the experimental research on high-harmonic generation in localized fields and, moreover, extend the prospects for solid-state high-harmonic generation. 


\subsection{High-Harmonic Generation and Imaging in Tailored Semiconductors}

Beyond enabling high harmonic generation from localized fields in plasmonic nanostructures, solids have an important advantage over gaseous targets: The structure and chemical composition of solid targets can be tailored via established microand nano-engineering methods. This enables novel means to shape the generated high-harmonic wave field in terms of intensity, phase or polarization. In turn, coherent signals generated in structured solids can be used for imaging and spectroscopy with nanometer spatial resolution.

Here, we use locally structured and chemically modified zinc oxide $(\mathrm{ZnO})$ and silicon targets to demonstrate high-harmonic generation and imaging [10]. Figure 19.2a depicts the experimental setup and principle. A zinc oxide crystal with an array of microcones milled to its surface (focused ion beam fabrication, see scanning electron micrograph in Fig. 19.2c) is placed in the focus of an infrared ( $2.1 \mu \mathrm{m}$ central wavelength), 70-femtosecond laser beam with $10 \mathrm{kHz}$ pulse repetition rate. At incident peak intensity exceeding $30 \mathrm{GW} \mathrm{cm}{ }^{-2}$, high-harmonic radiation up to the 9th harmonic order of the fundamental frequency (see Fig. 19.2b) is generated in the target. The high-harmonic generation is localized to sub-wavelength sized regions at the apexes of the cones due to a concentration of the incident laser light by total internal reflection and interference, leading to an at least 10 -fold intensity enhancement. The localized emission leads to a 2-dimensional diffraction pattern in the far-field, which is shown for the 3rd (red spots) and 5th (blue) harmonic far-field intensity in the inset in Fig. 19.2a.

At this point, we employ two approaches to get information on the intensity distribution at the sample plane. Since the wavelength of the 3rd and 5th harmonics are in the visible range, direct imaging using a high-numerical-aperture objective in combination with a bandpass filter and a charge-coupled device camera is possible. Figure 19.2d shows the directly imaged 5th harmonic pattern at the sample plane.

In order to illustrate that such an imaging approach is also transferable to harmonics with higher photon energies, where refractive optics are not available, we reconstruct the exit amplitude from the far-field intensity by applying a phase retrieval algorithm as described in Chap.18. The reconstructed amplitudes are shown in Fig. 19.2e. The field of view of the reconstruction is reduced due to limited spatial coherence and low signal-to-noise ratio of the diffraction pattern. However, the central spots clearly register to the arrangement of the cones in Fig. 19.2c, indicating the possibilities to image the nanoscale structures in solids.

In addition to structural modifications, which affect the driving laser field, local chemical changes also lead to enhanced high-harmonic generation in solid targets, as shown in Fig. 19.3 for the example of a gallium-ion-implanted silicon target. The 

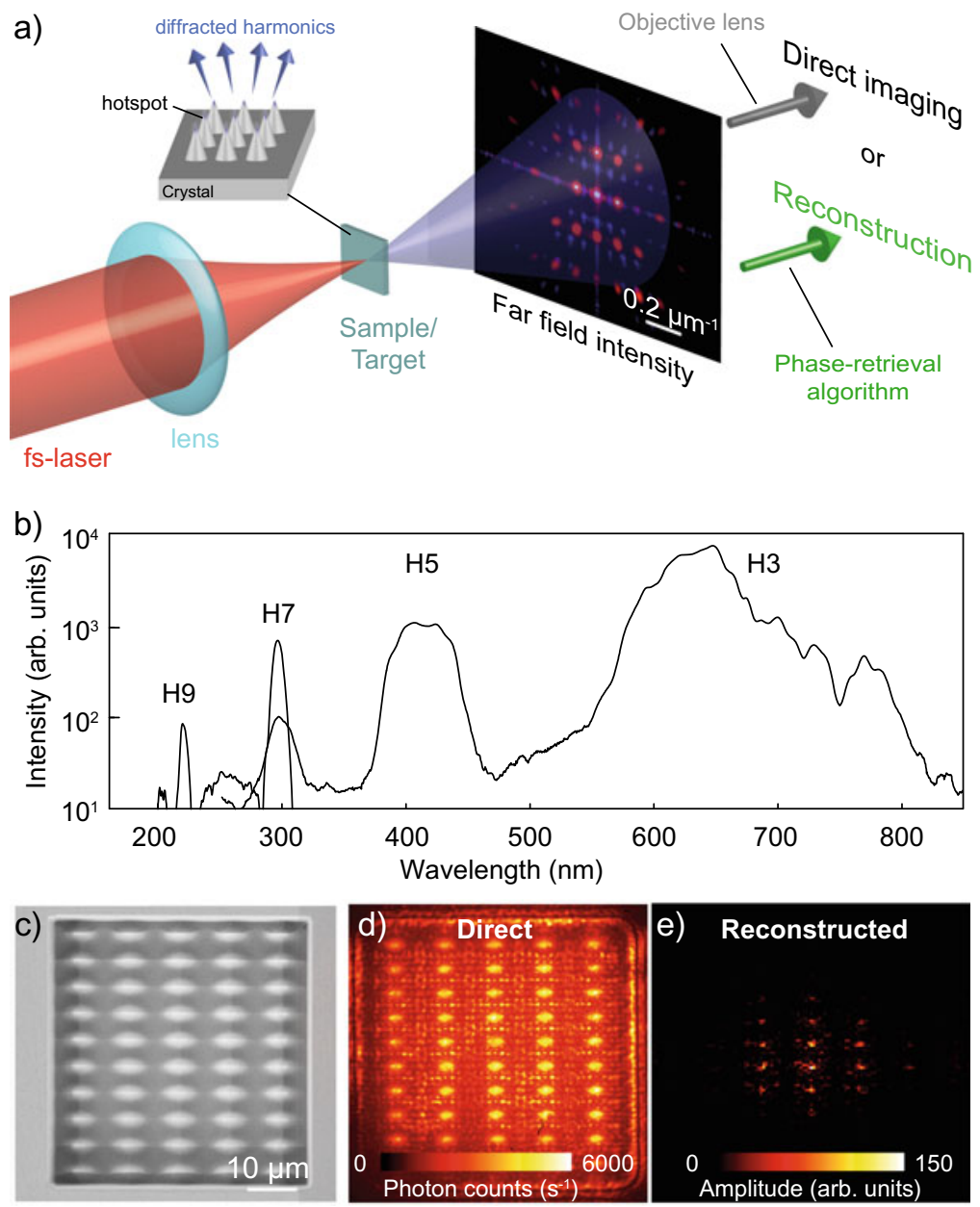

Fig. 19.2 High-harmonic generation in $\mathrm{ZnO}$ structures. a Schematic illustration of the experiment. The far-field intensity distribution, which is recorded with a 3-color CMOS sensor, shows diffracted third- (red) and fifth- (blue) harmonic signals emitted from a cone grating (see SEM image in c). The upper inset shows a 3-dimensional illustration of the microcones on the crystal's surface. b High-harmonic spectrum from a structured target illuminated with amplified 2.1- $\mu \mathrm{m}$ laser pulses. The harmonic orders are labeled H3-H9. Harmonics H7 and H9 were measured with a different spectrometer exhibiting a higher sensitivity in the spectral range below $350 \mathrm{~nm}$. c Scanning electron micrograph of a 2D-grating of microcones on $\mathrm{ZnO}$. d, e Comparison of the directly imaged fifth harmonic signal (Direct) using an objective and a phase-retrieval reconstruction of the exit amplitude (Reconstructed) using the far-field intensity 

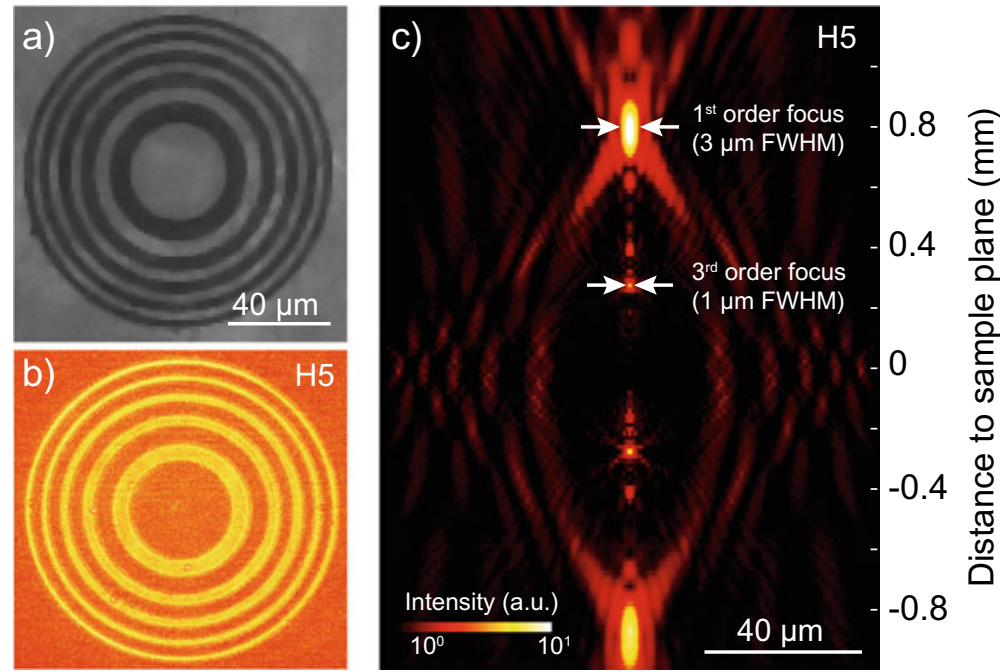

Fig. 19.3 Fresnel zone plate high harmonic source in silicon. a Scanning electron micrograph of a gallium-implanted Fresnel zone plate pattern (darker region) on a silicon wafer. b Intensity distribution of the fifth harmonic signal (H5, compare spectrum in Fig. 19.2b) at the sample plane. c Focus scan of imaging objective showing the azimuthally-integrated fifth-harmonic signal as a function of distance to the sample plane $(z=0)$

scanning electron microscope image in Fig. 19.3a shows the Fresnel zone plate pattern written into the silicon surface (dark regions were exposed to low-dose gallium ions). The gallium ions create defects in the silicon matrix, which lead to a local enhancement of the high-harmonic generation process, most likely by inducting mid-gap state in the silicon band structure. Figure $19.3 \mathrm{~b}$ is an image of the fifthharmonic emission at the sample plane, recorded with the objective lens, showing an enhanced signal in the gallium-implanted regions.

Generally, imaging of high-harmonic signals from locally structured and chemically modified solid targets represents a novel means to investigate local strong-field phenomena in condensed matter systems at nanometer scales, with potential capabilities for temporally or spectroscopically resolved studies. In turn, such sources also allow for the control of the generated high-harmonic wave field, as the Fresnel zone target in Fig. 19.3 illustrates.

The source leads to a focusing of the generated harmonic radiation to diffraction limited spot sizes. Figure 19.3c shows a focus scan of the azimuthally integrated intensities at different distances to the sample plane along the optical axis. Further results show that also the phase and polarization of the generated emission can be modified in such schemes.

In conclusion, our study demonstrates that strong-field effects are generally excitable in localized laser fields, e.g. by employing plasmonic nanostructures for field enhancement. However, gas-phase high harmonic generation lacks conversion 
efficiency in such scenarios due to a small number of gas atoms in the localized generation volume and the resulting insufficient coherent build-up of the high-harmonic signal. Instead of coherent high-harmonic emission, we observed bright incoherent extreme-ultraviolet fluorescence, which stems from multiphoton and strong-field excited and ionized gas atoms.

In contrast to gas-phase targets, in solid media an efficient generation of high harmonics in localized fields is possible. In structured $\mathrm{ZnO}$ and silicon targets we concentrated the driving laser field in microscopic cones and wedges and observed enhanced coherent high-harmonic emission from sub-wavelength sized generation volumes. In a second approach, we demonstrated that chemical modifications also influence the high-harmonic generation in solids. Direct imaging or phase-retrieval reconstruction of the localized coherent emission will enable new means to study strong-field phenomena in solid-state systems. Additionally, tailored solids will enable the development of new types of sources for tailored high-harmonic wave fields.

\section{References}

1. Kim, S., et al.: High-harmonic generation by resonant plasmon field enhancement. Nature 453(7196), 757-760 (2008). http://dx.doi.org/10.1038/nature07012

2. Park, I.Y., et al.: Plasmonic generation of ultrashort extreme-ultraviolet light pulses. Nat. Photonics 5(11), 677-681 (2011). https://doi.org/10.1038/nphoton.2011.258, http://www.nature. com/doifinder/10.1038/nphoton.2011.258

3. Sivis, M., Ropers, C.: Generation and bistability of a waveguide nanoplasma observed by enhanced extreme-ultraviolet fluorescence. Phys. Rev. Lett. 111(8), 085001 (2013). https:// doi.org/10.1103/PhysRevLett.111.085001, http://link.aps.org/doi/10.1103/PhysRevLett.111. 085001

4. Sivis, M., et al.: Nanostructure-enhanced atomic line emission. Nature 485(7397), E1E2 (2012). https://doi.org/10.1038/nature10978, http://www.nature.com/doifinder/10.1038/ nature10978, http://www.ncbi.nlm.nih.gov/pubmed/22575967

5. Sivis, M., et al.: Extreme-ultraviolet light generation in plasmonic nanostructures. Nat. Phys. 9(5), 304-309 (2013). https://doi.org/10.1038/nphys2590, http://www.nature.com/doifinder/ $10.1038 /$ nphys 2590

6. Raschke, M.B.: High-harmonic generation with plasmonics: feasible or unphysical? Ann. Phys. 525(3), A40-A42 (2013). https://doi.org/10.1002/andp.201300721, http://doi.wiley.com/10. 1002/andp.201300721

7. Han, S., et al.: High-harmonic generation by field enhanced femtosecond pulses in metalsapphire nanostructure. Nat. Commun. 7(May), 13105 (2016). https://doi.org/10.1038/ ncomms13105, http://www.nature.com/doifinder/10.1038/ncomms 13105

8. Vampa, G., et al.: Plasmon-enhanced high-harmonic generation from silicon. Nat. Phys. 13(7), 659-662 (2017). https://doi.org/10.1038/nphys4087

9. Imasaka, K., et al.: Antenna-enhanced high harmonic generation in a wide-bandgap semiconductor ZnO. Opt. Express 26(16), 21364 (2018). https://doi.org/10.1364/OE.26.021364, https://www.osapublishing.org/abstract.cfm?URI=oe-26-16-21364

10. Sivis, M., et al.: Tailored semiconductors for high-harmonic optoelectronics. Science 357, 6348 (2017). https://doi.org/10.1126/science.aan2395 
Open Access This chapter is licensed under the terms of the Creative Commons Attribution 4.0 International License (http://creativecommons.org/licenses/by/4.0/), which permits use, sharing, adaptation, distribution and reproduction in any medium or format, as long as you give appropriate credit to the original author(s) and the source, provide a link to the Creative Commons license and indicate if changes were made.

The images or other third party material in this chapter are included in the chapter's Creative Commons license, unless indicated otherwise in a credit line to the material. If material is not included in the chapter's Creative Commons license and your intended use is not permitted by statutory regulation or exceeds the permitted use, you will need to obtain permission directly from the copyright holder.

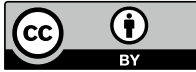

